\title{
Studying network mechanisms using intracranial stimulation in epileptic patients
}

\author{
Olivier David 1,2,3 ${ }^{\text {, Julien Bastin }}{ }^{1,2}$, Stéphan Chabardès ${ }^{1,2,4}$, Lorella Minotti ${ }^{1,2,5}$ and Philippe Kahane ${ }^{1,2,5,6}$ \\ INSERM U836, Grenoble Institut des Neurosciences, Grenoble, France \\ 2 Université Joseph Fourier, Grenoble, France \\ ${ }^{3}$ Clinique Universitaire de Neuroradiologie et Unité IRM, Centre Hospitalier Universitaire, Grenoble, France \\ ${ }^{4}$ Clinique Universitaire de Neurochirurgie, Centre Hospitalier Universitaire, Grenoble, France \\ ${ }^{5}$ Laboratoire de Neurophysiopathologie de l'Epilepsie, Clinique Universitaire de Neurologie, Centre Hospitalier Universitaire, Grenoble, France \\ ${ }^{6}$ CTRS-IDEE, Centre Hospitalier Universitaire, Lyon, France
}

Edited by:

Barry Horwitz, National Institutes of Health, USA

\section{Reviewed by:}

Sacha van Albada, Research Center

Jülich, Germany

Arpan Banerjee, Florida Atlantic

University, USA

\section{*Correspondence:}

Olivier David, Grenoble Institut des Neurosciences - INSERM U836,

Chemin Fortuné Ferrini - Bât EJ Safra, 38700 La Tronche, France.

e-mail: olivier.david@ujf-grenoble.fr
Patients suffering from focal drug-resistant epilepsy who are explored using intracranial electrodes allow to obtain data of exceptional value for studying brain dynamics in correlation with pathophysiological and cognitive processes. Direct electrical stimulation (DES) of cortical regions and axonal tracts in those patients elicits a number of very specific perceptual or behavioral responses, but also abnormal responses due to specific configurations of epileptic networks. Here, we review how anatomo-functional brain connectivity and epilepsy network mechanisms can be assessed from DES responses measured in patients. After a brief summary of mechanisms of action of brain electrical stimulation, we recall the conceptual framework for interpreting DES results in the context of brain connectivity and review how DES can be used for the characterization of functional networks, the identification of the seizure onset zone, the study of brain plasticity mechanisms, and the anticipation of epileptic seizures. This pool of exceptional data may be underexploited by fundamental research on brain connectivity and leaves much to be learned.

Keywords: neurostimulation, epilepsy, connectivity, networks, cognition, plasticity, electroencephalography

\section{INTRODUCTION}

Epilepsy is a common chronic neurological disorder characterized by recurrent spontaneous seizures showing paroxysmal electrical activity. A cortical imbalance between excitation and inhibition within local and large-scale networks is likely to trigger abnormal brain electrical activity and, thus, to be the pathophysiological basis for epilepsy. In models of epilepsy, either in vitro or in vivo, such imbalance can be induced in several ways. For instance, status epilepticus, i.e., seizures lasting more than $30 \mathrm{~min}$, can be initiated by blocking inhibition by injecting GABA receptors antagonists, e.g., bicuculline (Jefferys and Whittington, 1996). In the kindling model (Morimoto et al., 2004), permanent increases in seizure susceptibility can be provoked by repeated, although sparse, electrical stimulation of some brain sites.

Some patients with drug-resistant focal epilepsy in whom the epileptogenic zone cannot be defined with non-invasive measures are explored using depth electrodes (stereoelectroencephalography, SEEG) and/or subdural grids and strips (electrocorticography, ECoG) (Spencer et al., 2006). These techniques allow to obtain data of exceptional value for studying brain dynamics in correlation with pathophysiological (Jirsch et al., 2006) and cognitive (Jerbi et al., 2009) processes. In particular, they allow measuring intracranial functional responses to assess connectivity of the human brain beyond limitations of other techniques, such as post mortem dissections (Mesulam, 1979), diffusion tensor imaging (Jones, 2008), functional magnetic resonance imaging (Roebroeck et al., 2009), and combination of transcranial magnetic stimulation and neuroimaging (Paus et al., 1997).
Since the pioneering works of Penfield and Jasper (1954) performed during surgical interventions, it is also well known that direct electrical stimulation (DES) of cortical regions and axonal tracts allows a number of very specific perceptual or behavioral responses to be obtained. Despite the development of neuroimaging techniques, DES remains the gold standard for mapping brain functions, particularly regarding functional specialization whereas some interrogations remain when assessing functional integration (Mandonnet et al., 2010). For instance, DES of language areas performed during tumor resection induces very specific speech disturbances in awake patients (Duffau et al., 2008). Similarly, using subdural and/or depth electrodes implanted in epileptic patients for presurgical evaluations, various sensations, or behavioral effects elicited by DES have been reported (see below Section "Functional Mapping of the Eloquent Cortex Using DES"). In addition to these functional studies, several teams have used DES either to estimate functional anatomical connectivity (Buser and Bancaud, 1983; Matsumoto et al., 2004, 2007; Catenoix et al., 2005; Lacruz et al., 2007; Rosenberg et al., 2009), or to identify the epileptogenic area by searching for after-discharge thresholds (Cherlow et al., 1977; Engel et al., 1981; Chitoku et al., 2003) or abnormal brain responses (Valentin et al., 2002, 2005a,b; Flanagan et al., 2009), and by eliciting auras (Schulz et al., 1997) or complete seizures (Wieser et al., 1979; Bernier et al., 1987; Munari et al., 1993; David et al., 2008). These epileptic events induced by DES are likely to occur because epileptogenic networks may be particularly prone to short-term plasticity of synaptic weights induced by DES, at least in mesial temporal lobe epilepsy (Wilson et al., 1998; David et al., 2008). 
Direct electrical stimulation is thus a very powerful technique to investigate network mechanisms in epilepsy. Here, we will review how brain connectivity can be assessed from responses obtained after electrical stimulation of cortical regions in epileptic patients implanted with depth or subdural grids. We will cover neither animal studies, nor therapeutic brain stimulation (for review, see Saillet et al., 2009). After a brief summary of mechanisms of action of brain electrical stimulation, we will first recall the conceptual framework that is classically used in imaging neuroscience to study brain connectivity. It appears that DES studies do not fit easily in this interpretational context, because electrical stimuli are nonphysiological and elicit unusual neural responses. Second, we will briefly review studies that aimed at assessing the main brain functions using DES in epileptic patients. Third, abnormal responses to DES will be reviewed in the context of the identification of the seizure onset zone. Fourth, we will show how DES can be used to study brain plasticity. Finally, we will indicate how weak stimulation can also be tailored to anticipate seizures by using properties of brain responses to DES in relation to short-term structural changes of brain networks. From this review, we will conclude that this pool of exceptional data is underexploited by fundamental research on brain connectivity and leaves much to be learned.

\section{MECHANISMS OF ACTION OF BRAIN ELECTRICAL STIMULATION}

The effects of brain electrical stimulation within the central nervous system can be studied using neural modeling, neural recording, neurochemistry, and functional imaging (for review see McIntyre et al., 2004). Mechanisms of action of brain electrical stimulation are however not well understood because of the large number of intermingled processes that are initiated. Charge injection across the electrode/electrolyte (brain tissue) interface involves both capacitive and Faradaic mechanisms that interact and result in complex electrochemical reactions (Merrill et al., 2005). In view of this complexity, the choice of electrode material and geometry, and of stimulating patterns is crucial. One method that is commonly used for functional electrical stimulation of excitable tissue is the current-controlled method, in which a current source is attached between the working and counter electrode. Of considerable importance is the shape of current pulses. Specifically, it has been shown that monophasic pulses induce more tissue damage than biphasic pulses (Piallat et al., 2009), and should thus be avoided for long periods of stimulation. However, monophasic pulses are more efficient to initiate action potentials (Merrill et al., 2005), and are commonly used for short trains of stimulation in patients implanted with depth electrodes (Valentin et al., 2002, 2005a,b; Catenoix et al., 2005; Zumsteg et al., 2006a,b; David et al., 2008; Flanagan et al., 2009; Rosenberg et al., 2009). Biphasic pulses are nonetheless also used for acute stimulation by several teams (Wilson et al., 1990, 1998; Zangaladze et al., 2008; Jacobs et al., 2010).

It is now commonly accepted that the primary targets of intracerebral electrical stimulation are (large myelinated) axons, and not cell bodies (Nowak and Bullier, 1998a,b; Holsheimer et al., 2000; Kiss et al., 2003), and in particular that the initial segment of axons is the most excitable element recruited by electrical stimulation (Rattay, 1999). This has important implications for functional connectivity studies of DES responses. Indeed, both orthodromic and antidromic action potential propagation, which activates respectively the output and input structures of the stimulated area, can be observed. Also, one cannot completely exclude the activation of en passant fibers (axonal tracts), in particular when small deep nuclei are stimulated with strong currents. It is thus clear that localized microstimulation has remote effects, in addition to local ones. This is well documented from intracranial recordings that are specifically reviewed here, but also from functional imaging (Zonenshayn et al., 2000; Tolias et al., 2005; Canals et al., 2008).

Intracranial measurements of electrophysiological responses to DES are thought to capture mainly postsynaptic potentials resulting from indirect mono- or poly-synaptic cell activation (Jankowska et al., 1975) and thus provide a very interesting window on functional connectivity. However, there is an important limitation to this technique: DES delivers non-physiological stimuli and may activate pathways in an unusual way, e.g., antidromically. Therefore, because DES causes patterns of activation that may be different than natural neural activity, there will always be a potential indeterminacy in assessing true directionality of anatomo-functional connections identified with this technique. In other words, results of DES alone cannot be used to unequivocally establish that a given structure is naturally involved in a given function, despite DES of this structure has specific effects on that function. Empirically though, DES studies give invaluable insights into brain function and brain connectivity.

\section{CONCEPTS OF BRAIN CONNECTIVITY IN THE CONTEXT OF DES STUDIES}

Historically, behavioral consequences of localized brain lesions and biophysical measures of brain responses to various stimuli helped define what is now accepted as functional specialization of brain regions. This has led to localizationist theories of brain functions, but functional specialization is obviously not limited to location, because dense interregional connectivity makes any region of the brain part of an extended network (Sporns et al., 2005). Adhering to the principle of functional specialization thus does not necessarily imply that any function can be localized in a single area. Conversely, a single area may support different functions depending on its different connections.

For clarity, we will adopt the following definition:

Definition 1. A brain region participates in a brain function if a change in its activity is correlated to a change in that function, as assessed by subject's perception or behavior.

Note that this definition may be difficult to address experimentally if unconscious, or weakly observable, processes take place. In the context of DES studies, one could think of:

Corollary 1.1. A change in subject's perception or behavior induced by DES of a brain region indicates the functional specialization of that region.

This view of DES as a means to demonstrate functional specialization of specific brain regions predominates in the literature, and has proved to be very useful for functional neurosurgery of eloquent cortex. However, as perfectly reviewed in (Mandonnet et al., 2010), since each area responsive to stimulation is in fact an input gate into a large-scale network rather than an isolated discrete functional site, DES may lead to interpretations that a structure is crucial 
because of the induction of a transient functional response when stimulated, whereas this effect is caused by the backward spreading of neuronal waves to another essential area. This might explain, at least in part, why apparently similar electrically induced symptoms can be elicited by distant areas (Mulak et al., 2008). An important modification to Corollary 1.1 is therefore required:

Corollary 1.2. A change in subject's perception or behavior induced by DES of a brain region indicates the functional specialization of a large-scale network of which that region either is an input/output or a part.

Though the physical effects of DES are very focal $(<5 \mathrm{~mm})$, "physiological" effects of DES clearly suggest that functional specialization is only meaningful in the context of functional integration. In DES experiments, the stimulated point is only an input gate into a large distributed network. As suggested by others (Mandonnet et al., 2010), one should forget about distinct localizationist and connectionist models and shift toward a theory that integrates these two when analyzing how DES works.

Functional integration is quantified using measures of functional connectivity which can be thought of as an extension of functional specialization in which the activity of a brain region is no longer correlated to an extrinsic measure (behavior or perception), but to the activity of one, or more, other brain regions:

Definition 2. Two regions are functionally connected if their activities co-vary.

In the context of mechanisms of action of DES, this can be rephrased as:

Corollary 2.1. Brain regions showing specific responses to DES of a brain region are functionally connected to it, or send axonal tracts passing through this region.

Corollary 2.1 clearly indicates that there is an inherent indeterminacy in the interpretation of connectivity results from DES experiments, which may not appear at first sight of the standard Definition 2 of functional connectivity. This has to be kept in mind for the following of this review.

Note also that, according to Definition 2, functional connectivity depends on how brain activity is defined (raw local field potentials, firing rate, etc.) and also on the measure of correlation, or covariation, of this activity. Therefore, there are as many measures of functional connectivity as ways of defining covariations between variables (for reviews see Quian Quiroga et al., 2002; David et al., 2004; Wendling et al., 2009). Approaches can rely on linear or non-linear metrics, can be bivariate (e.g., simple linear correlation) or multivariate (e.g., multivariate autoregressive models), and can account for time delays if necessary. An important distinction concerns statistical models versus biophysical models. Statistical models consider brain time series as any time-embedded process, and compute standard measures of interactions. Historically, they clearly predominate. Biophysical models are marginal and recent in comparison, but have the interesting feature to give access to variables that are neuronally interpretable (e.g., synaptic efficacy). This class of models includes dynamic causal models (DCM) (Friston et al., 2003; David et al., 2006a).
In theory, functional connectivity does not necessitate anatomical connectivity. For instance, it has been shown in rat hippocampal slices bathing in low- $\mathrm{Ca}^{2+}$ solution that extracellular diffusion, most probably of potassium, was sufficient to synchronize anatomically unconnected populations of neurons and propagate low-frequency epileptiform activity (Lian et al., 2001). However, in the context of cortical electrical stimulation, we will assume functional connectivity is implicitly supported by anatomical connectivity, by the means of direct or indirect pathways.

\section{FUNCTIONAL MAPPING OF THE ELOQUENT CORTEX USING DES}

Direct electrical stimulation has been extensively used in epilepsy, and a few other neurological disorders such as Parkinson's disease, for mapping of normal functions in the human brain. For epilepsy resective surgery, the main goal of DES is to identify the eloquent cortex, i.e., crucial functional regions to be spared by surgery.

One must be careful when assessing the effect of DES of epileptic cortices, because of possible structural changes that may have occurred in the lead-up or in response to repeated seizures. However, not every part of the brain studied by intracerebral or subdural electrodes is pathological (Lachaux et al., 2003), and DES results in epileptic patients have revealed the same somatotopic organization of the primary sensori-motor areas as in healthy brains, as well as congruent findings with those coming from functional magnetic resonance imaging studies in normal volunteers (Lobel et al., 2001). However the physiological significance of DES should be questioned when the stimulation is applied inside or at the vicinity of an epileptogenic lesion, or when it elicits after-discharges, i.e., electroencephalographic seizures evoked in response to cortical stimulation.

Largely for historical reasons, the commonly applied method of stimulation for functional mapping consists of using 3-20 s trains of constant current $50-60 \mathrm{~Hz}$ biphasic square wave pulses of $0.2-1 \mathrm{~ms}$ duration, at current intensity $(\sim<10 \mathrm{~mA})$ set below the threshold for evoking after-discharges (Penfield and Jasper, 1954; Luders et al., 1986). High frequency stimulation elicits both positive behavioral response (e.g., muscle contraction) and negative response (e.g., speech arrest). However, a number of brain areas seem to be "silent" after DES, which does not mean lack of function of those regions because subtle cognitive or behavioral changes can be missed. Recently, it has been demonstrated that stimulating at low frequencies $(5-10 \mathrm{~Hz})$ may be as effective for functional mapping, with the significant benefit of reducing the tendency of electrical stimulation to produce after-discharges (Zangaladze et al., 2008). In contradiction, DES at lower frequencies $(1 \mathrm{~Hz})$ would be of special interest for eliciting seizures (Munari et al., 1993). Whatever the frequency used, the electrical field produced by DES depends on other stimulation parameters such as the pulse duration and current intensity, and bipolar DES produces a more localized current distribution than unipolar stimulation (Nathan et al., 1993).

Below, we will briefly review DES assessment of sensorimotor and language processing, which are the systems most commonly investigated by DES. In principle, numerous other processes can be examined, such as verbal memory (Ojemann, 2003), spatial cognition, experiential auras, mental rotation (Thiebaut de Schotten et al., 2005), limbic responses (Elliott et al., 2009), out-of-body 
experiences (Blanke et al., 2002), and dreamy state phenomena (Vignal et al., 2007). For completeness, note that an exhaustive review focused very recently on perceptual and behavioral phenomena induced by DES in human beings (Selimbeyoglu and Parvizi, 2010).

\section{SENSORIMOTOR PROCESSING}

The classic work of Penfield and Boldrey (1937) brought evidence in humans that cortical maps representing body parts (somatotopic maps) could be found in the primary motor and sensory cortices. In addition to body parts, it was proposed recently to map the motor cortex with DES as a function of behavioral repertoire (Graziano and Aflalo, 2007). The specific contribution to motor control of the different medial premotor areas was also evaluated using DES, including supplementary motor area (SMA), pre-SMA and cingulate motor area (CMA) (Lim et al., 1994; Luders et al., 1995; Chassagnon et al., 2008; Sumner et al., 2007). These studies particularly suggest that SMA and pre-SMA are strongly involved in motor inhibition. DES was also very useful to delineate human frontal eye fields in BA6 (Blanke et al., 2000; Lobel et al., 2001) and to examine cortico-spinal connectivity and muscle contraction during voluntary movement (Ikeda et al., 2002; Szelenyi et al., 2005).

Regarding sensory processing, early attempts discovered the possibility to obtain olfactory, gustatory, auditory, and somato-sensory responses to DES, Recently, the temporo-peri-Sylvian vestibular cortex was described in human from DES (Kahane et al., 2003) and DES of the insula was reported to be able to elicit pain (Afif et al., 2008). Visual responses to DES in the occipital lobe are common but vary according to stimulated areas and range from simple to complex visual form, color, and illusions (Lesser et al., 1998; Puce et al., 1999; Lee et al., 2000).

\section{LANGUAGE PROCESSING}

Direct electrical stimulation for language mapping in pharmacologically intractable epilepsy patients undergoing resection of epileptogenic cortex in the language dominant hemisphere has been widely and successfully used to diminish the probability of occurrence of language decline following the surgery. Because of response specificity, the tasks chosen to evaluate the effect of DES on language processing are critical. For instance, DES may induce speech arrest during counting in only $1 / 3$ of all positive sites (i.e., sites where DES affected task performance) identified using other tasks such as naming or reading (Hamberger, 2007). It is therefore important to test in each patient several tasks, which incidentally makes DES very useful to identify individual components of language processing and their neural correlates. Hence, tasks such as visual or auditory object naming, automatic speech (e.g., counting), writing and reading were used to map language functions distributed in the frontal-temporal-parietal language network. Word retrieval was associated with posterior temporal lobe stimulated sites (Hamberger, 2007; Mani et al., 2008), whereas more anterior sites were involved in semantic processes per se (Sharp et al., 2004; Boatman and Miglioretti, 2005; Trebuchon-Da Fonseca et al., 2009) and speech production (Afif et al., 2010).

\section{DES AS A TOOL FOR REVEALING NEUROANATOMICAL PATHWAYS}

The vast majority of literature devoted to functional connectivity studies using cortical electrical stimulation in epileptic patients (Wilson et al., 1990; Catenoix et al., 2005; Lacruz et al., 2007; Rosenberg et al., 2009) considers DES as an efficient way to estimate which brain regions are anatomically connected to the site of stimulation. Responses to DES usually consist of a sharp deflection followed by a slow wave (Lacruz et al., 2007). Estimation of neuroanatomical pathways is performed by (i) the detection of sites showing such a response to the stimulation (by looking at amplitude variations above a threshold defined according to baseline level); (ii) the measure of first peak latency to estimate roughly whether anatomical connections are direct or indirect. Methodology is thus typically limited to quantifying amplitude and delay of DESevoked responses. Our recent study, discussed in a following section (David et al., 2008), went beyond this by using biophysical modeling of intracranial data to assess brain connectivity from responses to DES.

Because patients suffering from mesial-temporal lobe epilepsy are good candidates for resective surgery, they are commonly explored with depth electrodes, located in temporal limbic regions and also in suspected regions of seizure propagation such as temporal, insular, and frontal neocortex. As a consequence, functional anatomical connectivity of the temporal and of the frontal lobes is extensively discussed in the DES literature.

In Wilson et al. (1990), connections within the human mesial temporal lobe were investigated using brief, single pulses of electric stimulation of 8 different limbic areas in 74 epileptic patients. Biphasic, rectangular pulses of $100 \mu$ s/phase duration were delivered at a rate of $0.1 \mathrm{~Hz}$ or less, with currents ranging from 0.25 to $5.0 \mathrm{~mA}$. Specific measures included response probability, amplitude, latency (first peak detection) and conduction velocities (estimated distance between targeted stimulation and recording site divided by the onset response latency). Evoked responses were obtained in amygdala, entorhinal cortex, presubiculum, hippocampus, and parahippocampal gyrus. The authors were able to differentiate fast pathways (perforant path connecting entorhinal cortex to anterior hippocampus; $4.4 \mathrm{~ms}$ conduction delay and $3.64 \mathrm{~m} / \mathrm{s}$ conduction speed) from slow pathways (connection between amygdala and middle hippocampus; $24.8 \mathrm{~ms}$ and $0.88 \mathrm{~m} / \mathrm{s}$ ). In addition, from the absence of contralateral responses to the stimulation of the mesial temporal structures, they did not find a functional contralimbic projection in the human brain, which appears consistent with reduced hippocampal commissural connections in lower primates as compared to lower animals.

In (Lacruz et al., 2007), functional anatomical connectivity between frontal (medial, lateral, and orbital) and temporal (entorhinal, medial, and lateral) lobes has been described extensively using brain responses to $1 \mathrm{~ms}$ single pulses, delivered every $10 \mathrm{~s}$ at current intensity ranging between 4 and $8 \mathrm{~mA}$, obtained in 51 epileptic patients implanted with subdural and depth electrodes. The measured variables were the presence or absence of early responses at different recording sites when stimulating each location and the latency of contralateral responses. Anatomical functional connections were assessed by quantifying the number of 
electrodes showing evoked responses. Contralateral temporal connections were found to be rare ( $<9 \%$ of stimulated hemispheres), as in (Wilson et al., 1990). This appeared distinct to interhemispheric frontal connections, which were faster and more common $(>57 \%$ of stimulated hemispheres). Intralobar connections were frequent, either in the temporal ( $>67 \%$ of stimulated hemispheres) or in the frontal ( $>78 \%$ of stimulated hemispheres) cortices. In addition, ipsilateral connections from frontal toward temporal cortices were found to be more prominent than in the opposite direction. Because of limited spatial sampling, but also of physiological limitations such as attenuation of the signal in polysynaptic pathways and the recruitment of inhibition, these connectivity figures should be regarded as the lower limit of the true functional connectivity.

Using single pulses ( $3 \mathrm{~mA}$ current intensity, $1 \mathrm{~ms}$ duration, $0.2 \mathrm{~Hz}$ frequency), Catenoix et al. (2005) were the first to describe orbitofrontal cortex responses following electrical stimulation of temporomesial structures in humans, in a small group of three patients. They found a long latency of the main response (222 ms on average) that suggested a polysynaptic projection of the hippocampus to the orbitofrontal cortex. In the absence of strong hippocampal commissural pathway in humans (Wilson et al., 1990, 1991), these results strongly support the existence of a temporal frontal pathway for the interhemispheric propagation of mesial temporal seizures (Lieb et al., 1991).

Using the same stimulation protocol, this team recently published an extension of this work on temporal frontal connections, where the focus was on reciprocal thalamocortical connectivity of the medial pulvinar (PuM) estimated in seven epileptic patients implanted with thalamic and cortical electrodes (Rosenberg et al., 2009). Cortical-evoked potentials to PuM stimulation were recorded from all explored cortical regions, except striate cortex, anterior cingulate, and postcentral gyrus. Response rate was high, and ranged from $80 \%$ in temporal neocortex, temporoparietal junction, insula, and frontoparietal opercular cortex to $34 \%$ in mesial temporal regions. Reciprocally, PuM responses were observed following cortical stimulation, with response rate ranging from 14\% (insula and frontoparietal opercular cortex) to $76-80 \%$ (temporal neocortex and mesial temporal regions). From these findings, it is clear that there exist functional pathways between thalamic medial pulvinar nucleus and numerous cortical regions, with preferential and fastest pathways that interconnect the PuM with the temporal neocortex, the temporoparietal junction and the insulo-opercular region. This explains why PuM is involved in most of temporal and insular lobe seizures (Rosenberg et al., 2006). These results agree with data gathered in non-human primates, except for the important connectivity between pulvinar and hippocampus in primates. Another important point is the unexpected imbalance between some reciprocal pathways (with the insula, notably), which could not be found using magnetic resonance tractography for instance (Behrens et al., 2003), whereas the connections between the major sensory thalamic relay nuclei and cortex are considered as "reciprocal" (Steriade et al., 1997).

\section{DES AS A TOOL FOR REVEALING EPILEPTOGENIC NETWORKS}

Single pulse (Valentin et al., 2002, 2005a,b; Flanagan et al., 2009), paired pulse (Wilson et al., 1998), and repetitive (i.e., trains of pulses) (Buser and Bancaud, 1983; Kahane et al., 1993, 2004; Landré et al., 2004; Kalitzin et al., 2005) stimulation have been shown to be useful in localizing the seizure onset zone in patients suffering from various forms of focal epilepsy.

\section{EPILEPTIC RESPONSES TO SINGLE PULSES OR TO SHORT TRAINS OF PULSES}

In a series of papers (Valentin et al., 2002, 2005a,b; Flanagan et al., 2009), Alarcón and colleagues classified responses to single pulses into three types: (i) "early responses" composed of a sharp wave often followed by a slow wave, which is ubiquitous and reflects normal processes. This is the type of response exploited for anatomical functional studies and discussed so far in this review; (ii) "delayed responses" that resembles an epileptiform discharge occurring later than $100 \mathrm{~ms}$ after stimulus, thus indicating polysynaptic processes. They are thought of as pathological responses generated by areas of seizure onset; (iii) "repetitive responses" that look like early responses, but with longer lasting oscillations which may be initiated by reverberating loops. In fact, they are similar to damped oscillations that can be obtained with neural mass models with connectivity parameters tuned so as to be just below the threshold of stability, e.g., with strong intrinsic excitatory efficacy or feedback connections (David et al., 2005).

Recently, Gotman and colleagues studied the effects of another standard type of stimulation, i.e., short trains of high frequency pulses, in patients having either mesiotemporal or neocortical seizure onset zones (Jacobs et al., 2010). They found that the sites showing interictal high frequency oscillations (HFOs - ripples, $80-250 \mathrm{~Hz}$; fast ripples, $250-500 \mathrm{~Hz}$ ), thought to be linked to seizure onset (Jirsch et al., 2006), were the same as those showing afterdischarges or seizures elicited by electrical stimulation (bipolar biphasic $60 \mathrm{~Hz}$ stimulus, pulse width $=0.5 \mathrm{~ms}, 3-4 \mathrm{~s}$ duration, electrical currents $<2 \mathrm{~mA}$ in mesial temporal and $<10 \mathrm{~mA}$ in neocortical regions), especially in neocortical regions.

\section{PLASTICITY OF EPILEPTOGENIC NETWORKS INDUCED BY REPEATED ELECTRICAL STIMULATION}

Delayed, repetitive and HFO responses directly point toward the idea that epileptogenic networks are hyperexcitable. In addition, there is evidence that epileptogenic networks are prone to exhibit fast structural modifications via the reorganization of synaptic weights: From in vitro and in vivo animal studies, it is well known that repeated electrical stimulation may induce massive reorganization of brain networks, particularly in the hippocampus (Bliss and Lomo, 1973). In humans, although long-term potentiation cannot be easily demonstrated because of lack of experimental possibilities, it has been possible to identify short-term plasticity effects.

The most standard, but not unique, paradigm of stimulation to study short-term plasticity is paired-pulse stimulation. Paired-pulse stimulation detects excitability changes on the assumption that the first (conditioning) stimulus acts to recruit recurrent excitatory or inhibitory activity, which affords detecting facilitatory or suppressive effect upon the response to the second (test) stimulus. Paired-pulse inhibition is thought to reflect changes of presynaptic release probability and the influence of recurrent inhibition (Zucker and Regehr, 2002). Analyzing the different patterns of paired-pulse facilitation and paired-pulse inhibition may also give 
some insights for distinguishing neuronal drivers from neuronal modulators (Reichova and Sherman, 2004). In mesial temporal lobe epilepsy (MTLE), paired-pulse induced changes of synaptic and intrinsic excitability are usually more easily observed in the hippocampus than in the neocortex (Koch et al., 2005). In vitro experiments (Feng et al., 2003) allowed to gather more information about neuronal mechanisms involved. Modulation of NMDA or kainate receptors, the receptor type depending on the structures involved, was systematically found to be associated with electrically induced short-term plasticity in epilepsy. As shown by using trains of stimuli at different frequencies (Feng et al., 2003; Schiller and Bankirer, 2007), short-term plasticity is frequency-dependent and can be either inhibitory or excitatory. When repetitive stimulation has an anti-epileptic effect, this effect is mediated mainly by shortterm synaptic depression of excitatory neurotransmission (Schiller and Bankirer, 2007).

In MTLE patients, in vivo, a significantly greater paired-pulse suppression has been observed in epileptic regions, by comparing the epileptic hemisphere to the contralateral intact hemisphere in 20 patients (Wilson et al., 1998). Hippocampal pathways and perforant path responses located in the epileptogenic lobe showed greater paired-pulse suppression of population post-synaptic potentials. These authors derived from these responses interesting hypotheses about adaptive enhanced inhibition to inhibit seizures produced by abnormal recurrent excitatory circuits. In other words, increased short-term plasticity has been interpreted as a functional consequence of the formation of abnormal recurrent inhibitory and excitatory pathways in the sclerotic hippocampus.

By analyzing the preictal modulation in time of the shape of responses evoked by $1 \mathrm{~Hz}$ stimulation in twenty MTLE patients, we have shown that the seizure onset zone was particularly likely to show fast changing evoked responses to DES, even of remote areas (David et al., 2008). In this study, we have first developed a pure data-driven analysis to illustrate that short-term plasticity could be quantified easily for clinical purposes. However, this type of data analysis, which is commonly used in DES studies, is very limited for the explanation of the underlying neural mechanisms. In a second step, we moved further and showed how synaptic plasticity could be explicitly modeled using generative models of local field potentials (David et al., 2005). We used dynamic causal modeling (DCM) (David et al., 2006a) and assumed different possible neural network configurations within the temporal lobe, with some connections expressing synaptic plasticity via a modulation of their weight between successive stimulations. After parameter estimation (intrinsic neural parameters and coupling between regions) from measured evoked responses and Bayesian model selection (Penny et al., 2004), we were able to estimate the general architecture of stimulated temporal networks, and to demonstrate that observed modification of the shape of evoked responses to DES was sufficiently explained by a modulation of excitatory efficacies within temporal lobe circuitry.

For instance, in two patients stimulated in the amygdala who showed a strong increase of the amplitude of responses in the anterior hippocampus a few stimulations before the seizure onset, an increase of effective connectivity between the temporal pole and the anterior hippocampus was found. This pathway is supported by human anatomical studies showing that the hippocampus receives afferents from all over the anterior temporal cortex and an amygdala-temporal fascicle that originates at the rostrolateral surface of the amygdala and specifically connects to the temporal pole (Klingler and Gloor, 1960; Chabardes et al., 2002). Furthermore, it is known from visual analysis of SEEG recordings that the temporal pole is a determinant structure, concomitantly with the hippocampus, during the onset of seizures in temporal lobe epilepsy in many patients (Kahane et al., 2002; Chabardes et al., 2005). The results obtained from this small DCM study call for an interpretation of the genesis of a certain type of mesial temporal lobe epilepsy as a preictal increase of the hippocampal afferents coming from the temporal pole, which could be triggered by the amygdala. This putative type of mesial temporal lobe epilepsy remains to be fully characterized.

It is important to develop further models of DES to better understand the network mechanisms of action of this type of stimulation. Among the different lines of research, a possibility is to extend the current DCM framework (Daunizeau et al., 2009) and, under due consideration of the limits of statistical inversion, represent different neurobiological mechanisms of synaptic plasticity more explicitly, such that their relative importance can be disambiguated by model selection. Since aberrant plasticity is a central pathological mechanism in many brain diseases, and particularly epilepsy, developing plastic DCMs that can distinguish between different aspects of synaptic plasticity has an interesting potential for establishing physiologically interpretable diagnostic markers. As an academic exercise, we have made an early attempt in this direction by proposing a reinterpretation of short-term plasticity induced by $1 \mathrm{~Hz}$ DES in the context of autopoietic theory (Varela et al., 1974; David, 2007). This deserves to be pursued.

\section{ACTIVE ANTICIPATION OF SEIZURE USING DES}

Brain networks are very complex and generate spontaneous brain dynamics that are difficult to understand fully. In the recent years, emphasis has been put on synchronous oscillations which have been proposed to support large scale integration during cognitive processes (Varela et al., 2001). Another interpretation of transient features of brain dynamics correlated to behavior, or thoughts, has generalized and extended frequency-specific synchronization using tools from non-linear physics that embed brain signals in a multidimensional "state space." Successive transient synchronization of brain activity may then be thought of as the evolution of the system through a temporal sequence of different local attractors segregated in that state space (Tsuda, 2001). This latter theoretical framework is interesting for reading epileptic signals because a seizure is characterized by hypersynchronous and non-linear oscillations, two properties that are well captured by the formalism of non-linear dynamics. Briefly, transient hypersynchronicity corresponds to the transition of the state of the system from a region of high-dimensional chaos toward a low-dimensional attractor, the shape of which depends on the kind of non-linearity of the signals. Thus a seizure can be interpreted as a transition of the brain states from a succession of high-dimensional "physiological" states toward a lowdimensional "epileptic" state. It is important to question what sorts of dynamics govern that transition. 
In the previous sections, we have shown that repetitive stimulation using strong current intensity $(>1 \mathrm{~mA})$ was able to initiate after-discharges and complete seizures (David et al., 2008), possibly by the means of short-term plasticity mechanisms in brain networks. These seizures then represent transitions due to shifts in one or more parameters of the system. These parameters, e.g., synaptic efficacies, are structural in the sense that they govern dynamic responses of the brain, e.g., evoked responses to a brief stimulation (David et al., 2006b). If the preictal modifications of the parameters show a slow time evolution which can take several minutes, hours or days, analyzing dynamical properties of the brain to a stimulation is a way to reveal structural modifications. In principle, it can thus be used to anticipate seizures.

Stimulation-based seizure anticipation, reviewed in (Kalitzin et al., 2010), has been proposed by Lopes da Silva and colleagues. Early trials were based on photic stimulation in patients having photosensitive epilepsy (Kalitzin et al., 2002; Parra et al., 2003). This was followed by an experimental validation using intracerebral stimulation of the hippocampus in six patients (Kalitzin et al., 2005) and by simulations (Suffczynski et al., 2008). They used intermittent pulse stimulation $(0.5-1 \mathrm{~mA} ; 0.1 \mathrm{~ms}$ pulse width; trains of $5 \mathrm{~s}$ duration) in the frequency range $10-20 \mathrm{~Hz}$ and quantified the responsiveness of neural networks using a quantitative measure of spectral phase demodulation called the relative phase clustering index (rPCI). Although seizures could not be anticipated precisely, regions showing high interictal rPCI correlated with the seizure onset zone. Measurements of the modulation of neural synchronization of brain networks by the means of electrical stimulation are thus potentially an interesting approach for seizure anticipation techniques. More clinical trials are needed to better evaluate the specificity of such findings.

\section{CONCLUSION}

In this review, we have tried to show the multiple possibilities of DES to study network mechanisms in epilepsy. They mainly relate to the opportunity to identify, in the human brain, epileptogenic and plastic network properties, in addition to oriented neuroanatomical pathways, which is unique. Strikingly, these exceptional data have not yet been the object of many methodological studies, possibly, at least in part, because of ethical aspects that cannot be underestimated. This let us suppose that many interesting features regarding brain connectivity remain to be discovered from DES data, should adequate signal analysis techniques be applied to them, and experimental protocols be not deleterious for the patients.

Though we have not reviewed extensively this issue here, there are actually very few DES studies that combine direct stimulation and recordings of cortical activity during fluctuations of resting state, cognitive tasks and/or behavioral evaluations (e.g., Sinai et al., 2009; see for review Selimbeyoglu and Parvizi, 2010). Among them, an attempt was made to decipher brain connectivity in an epileptic patient during vivid memory recollection following perirhinal DES (Barbeau et al., 2005). This study showed that there was a widespread (between limbic and visual brain areas) theta synchronization quantified using cross-correlation analyzes. Another recent advance on DES mechanisms and their relationship to local physiological brain states was made (Lesser et al., 2008). These authors showed that spontaneous brain electrical activity at the moment of DES delivery could be predictive of an after-discharge occurrence that was often restricted to sites belonging to the same functional network (motor, sensory, or language) as the site of stimulation. Therefore, these authors speculated that similar rapid variations may occur during normal intracortical communication and may underlie changes in the cortical organization of functions. A potentially very interesting line of research for the coming years would thus be exploit further this hypothesis and to examine (i) how the perceptual and behavioral effects of DES may vary according to the undergoing cognitive task, (ii) how functional connectivity is affected after DES is applied during various cognitive tasks and (iii) how resting state connectivity at the moment of DES delivery might predict DES effects, both at the behavioral and electrophysiological levels.

Because they allow transient modifications of brain dynamics, DES studies can investigate short-term plastic network properties of several systems in a limited amount of time. Under the hypothesis that epileptogenic networks are hyperexcitable, and thus prone to exhibit short-term plasticity, plasticity analysis of DES responses is potentially a very powerful clinical tool to quickly identify epileptic foci (David et al., 2008). Thus, DES studies go well beyond lesion studies performed in animals or in stroke patients. However, they present some important limitations for connectivity analyses: though perceptual, behavioral, and epileptic phenomena can easily be induced by DES in human beings (Kahane et al., 2004; Selimbeyoglu and Parvizi, 2010), the use of macroelectrodes precludes the precise control of the mechanisms by which neurologists or neurosurgeons interact with patients' brain. For instance, it is difficult to evaluate in what exact proportions antidromic and orthodromic propagation take place, or if observed effects are mainly mediated by the activation of local neuronal populations, or by the stimulation of en passant fibers that target distributed remote regions. These experimental limitations are crucial regarding the key concepts of functional brain mapping that underlie the way we analyze recorded data (see above Section "Concepts of Brain Connectivity in the Context of DES Studies"). In that respect, DES studies differ from other functional studies because they elicit unusual brain responses mediated by the means of unusual input gates to the human brain. This is something that has to be kept in mind when anatomical, functional, or computational models of the brain are derived from the DES literature. In terms of biophysical modeling of DES responses, the experimental uncertainty summarized in Corollary 2.1 , i.e., in a given experiment does DES target local neuronal populations or en passant fibers?, must be taken into account. For instance in the framework of DCM, this could correspond to loose priors on how DES input perturb the system, i.e., on the extrinsic connectivity matrix (David et al., 2008). Hopefully, in addition to these theoretical considerations, new data coming from the optogenetic literature in animals (Miesenbock, 2009) will shed new light on how one can model human brain connectivity from DES responses.

\section{ACKNOWLEDGMENTS}

This work was funded by INSERM, Agence Nationale pour la Recherche and Fondation pour la Recherche Médicale. 


\section{REFERENCES}

Afif, A., Hoffmann, D., Minotti, L., Benabid, A. L., and Kahane, P. (2008). Middle short gyrus of the insula implicated in pain processing. Pain $138,546-555$.

Afif, A., Minotti, L., Kahane, P., and Hoffmann, D. (2010). Middle short gyrus of the insula implicated in speech production: intracerebral electric stimulation of patients with epilepsy. Epilepsia 51, 206-213.

Barbeau, E., Wendling, F., Regis, J., Duncan, R., Poncet, M., Chauvel, P., and Bartolomei, F. (2005). Recollection of vivid memories after perirhinal region stimulations: synchronization in the theta range of spatially distributed brain areas. Neuropsychologia 43 , 1329-1337.

Behrens, T. E., Johansen-Berg, H., Woolrich, M. W., Smith, S. M., Wheeler-Kingshott, C.A., Boulby, P.A. Barker, G. J., Sillery, E. L., Sheehan, K., Ciccarelli, O., Thompson, A. J., Brady, J. M., and Matthews, P. M. (2003). Non-invasive mapping of connections between human thalamus and cortex using diffusion imaging. Nat. Neurosci. 6, 750-757.

Bernier, G. P., Saint-Hilaire, J. M., Giard, N., Bouvier, G., and Mercier,M. (1987). "Commentary: intracranial electrical stimulation," in Surgical Treatment of the Epilepsies, ed.J.Engel Jr. (New York: Raven Press), 323-334.

Blanke, O., Ortigue, S., Landis, T., and Seeck, M. (2002). Stimulating illusory own-body perceptions. Nature 419, 269-270.

Blanke, O., Spinelli, L., Thut, G., Michel, C. M., Perrig, S., Landis, T., and Seeck, M. (2000). Location of the human frontal eye field as defined by electrical cortical stimulation: anatomical, functional and electrophysiological characteristics. Neuroreport 11, 1907-1913.

Bliss, T. V., and Lomo, T. (1973). Longlasting potentiation of synaptic transmission in the dentate area of the anaesthetized rabbit following stimulation of the perforant path. J. Physiol. 232, 331-356.

Boatman, D. F., and Miglioretti, D. L. (2005). Cortical sites critical for speech discrimination in normal and impaired listeners. J. Neurosci. 25, 5475-5480.

Buser,P., and Bancaud, J. (1983). Unilateral connections between amygdala and hippocampus in man. A study of epileptic patients with depth electrodes. Electroencephalogr. Clin. Neurophysiol. $55,1-12$

Canals, S., Beyerlein, M., Murayama, Y., and Logothetis, N. K. (2008). Electric stimulation fMRI of the perforant pathway to the rat hippocampus. Magn. Reson. Imaging 26, 978-986.

Catenoix, H., Magnin, M., Guenot, M., Isnard, J., Mauguiere, F., and Ryvlin, P. (2005). Hippocampal-orbitofrontal connectivity in human: an electrical stimulation study. Clin. Neurophysiol. 116, 1779-1784.

Chabardes, S., Kahane, P., Minotti, L., Hoffmann, D., and Benabid, A. L. (2002). Anatomy of the temporal pole region. Epileptic. Disord. 4(Suppl. 1), S9-S15.

Chabardes, S., Kahane, P., Minotti, L., Tassi, L., Grand, S., Hoffmann, D., and Benabid, A. L. (2005). The temporopolar cortex plays a pivotal role in temporal lobe seizures. Brain 128, 1818-1831.

Chassagnon, S., Minotti, L., Kremer, S. Hoffmann, D., and Kahane, P. (2008). Somatosensory, motor, and reaching/ grasping responses to direct electrical stimulation of the human cingulate motor areas. J. Neurosurg. 109, 593-604.

Cherlow, D. G., Dymond, A. M., Crandall, P. H., Walter, R. D., and Serafetinides, E. A. (1977). Evoked response and after-discharge thresholds to electrical stimulation in temporal lobe epileptics. Arch. Neurol. 34, 527-531.

Chitoku, S., Otsubo, H., Harada, Y., Jay, V., Rutka, J. T., Weiss, S. K., Elliott, I., Ochi, A., Kitayama, M., and Snead, O. C. III (2003). Characteristics of prolonged afterdischarges in children with malformations of cortical development. J. Child Neurol. 18, 247-253.

Daunizeau, J., David, O., and Stephan, K. E. (2009). Dynamic causal modelling: a critical review of the biophysical and statistical foundations. Neuroimage doi: 10.1016/j.neuroimage.2009.11.062.

David, O. (2007). Dynamic causal models and autopoietic systems. Biol. Res. 40, 487-502.

David, O., Cosmelli, D., and Friston, K. J. (2004). Evaluation of different measures of functional connectivity using a neural mass model. Neuroimage 21 , 659-673.

David, O., Harrison, L., and Friston, K. J. (2005). Modelling event-related responses in the brain. Neuroimage $25,756-770$.

David, O., Kiebel, S. J., Harrison, L. M., Mattout, J., Kilner, J. M., and Friston, K. J. (2006a). Dynamic causal modeling of evoked responses in EEG and MEG. Neuroimage 30, 1255-1272.

David, O., Kilner, J. M., and Friston, K. J. (2006b). Mechanisms of evoked and induced responses in MEG/EEG. Neuroimage 31, 1580-1591.

David, O., Wozniak, A., Minotti, L., and Kahane, P. (2008). Preictal short-term plasticity induced by intracerebral 1
$\mathrm{Hz}$ stimulation. Neuroimage 39 1633-1646.

Duffau, H., Peggy Gatignol, S. T., Mandonnet, E., Capelle, L., and Taillandier, L. (2008). Intraoperative subcortical stimulation mapping of language pathways in a consecutive series of 115 patients with Grade II glioma in the left dominant hemisphere. J. Neurosurg. 109, 461-471.

Elliott, B., Joyce, E., and Shorvon, S. (2009). Delusions, illusions and hallucinations in epilepsy: 1.Elementary phenomena. Epilepsy Res. 85, 162-171.

Engel, J. Jr., Rausch, R., Lieb, J. P., Kuhl, D. E., and Crandall, P. H. (1981) Correlation of criteria used for localizing epileptic foci in patients considered for surgical therapy of epilepsy. Ann. Neurol. 9, 215-224.

Feng, L., Molnar, P., and Nadler, J. V. (2003). Short-term frequencydependent plasticity at recurrent mossy fiber synapses of the epileptic brain. J. Neurosci. 23, 5381-5390.

Flanagan, D., Valentin, A., Garcia Seoane, J. J., Alarcon, G., and Boyd, S. G. (2009) Single-pulse electrical stimulation helps to identify epileptogenic cortex in children. Epilepsia 50, 1793-1803.

Friston, K. J., Harrison, L., and Penny, W. (2003). Dynamic causal modelling. Neuroimage 19, 1273-1302.

Graziano, M. S., and Aflalo, T. N. (2007) Mapping behavioral repertoire onto the cortex. Neuron 56, 239-251.

Hamberger,M.J. (2007). Cortical language mapping in epilepsy: a critical review. Neuropsychol. Rev. 17, 477-489.

Holsheimer, J., Demeulemeester, H. Nuttin, B., and de Sutter, P. (2000) Identification of the target neuronal elements in electrical deep brain stimulation. Eur. J. Neurosci. 12, 4573-4577.

Ikeda, A., Miyamoto, S., and Shibasaki, H. (2002). Cortical motor mapping in epilepsy patients: information from subdural electrodes in presurgical evaluation. Epilepsia 43(Suppl. 9), 56-60.

Jacobs, J., Zijlmans, M., Zelmann, R., Olivier, A., Hall, J., Gotman, J., and Dubeau, F. (2010). Value of electrical stimulation and high frequency oscillations $(80-500 \mathrm{~Hz})$ in identifying epileptogenic areas during intracranial EEG recordings. Epilepsia 51, 573-582.

Jankowska, E., Padel, Y., and Tanaka, R. (1975). The mode of activation of pyramidal tract cells by intracortica stimuli. J. Physiol. 249, 617-636.

Jefferys, J. G., and Whittington, M. A. (1996). Review of the role of inhibitory neurons in chronic epileptic foci induced by intracerebral tetanus toxin. Epilepsy Res. 26, 59-66.
Jerbi, K., Ossandon, T., Hamame, C. M., Senova, S., Dalal, S. S., Jung, J., Minotti, L., Bertrand, O., Berthoz, A. Kahane, P., and Lachaux, J. P. (2009). Task-related gamma-band dynamics from an intracerebral perspective: review and implications for surface EEG and MEG. Hum. Brain Mapp. 30, 1758-1771.

Jirsch, J. D., Urrestarazu, E., LeVan, P., Olivier, A., Dubeau, F., and Gotman, J. (2006). High-frequency oscillations during human focal seizures. Brain 129, 1593-1608.

Jones, D. K. (2008). Studying connections in the living human brain with diffusion MRI. Cortex 44, 936-952.

Kahane, P., Chabardes, S., Minotti, L., Hoffmann, D., Benabid, A. L., and Munari, C. (2002). The role of the temporal pole in the genesis of temporal lobe seizures. Epileptic Disord. 4(Suppl. 1), S51-S58.

Kahane, P., Hoffmann, D., Minotti, L., and Berthoz, A. (2003). Reappraisal of the human vestibular cortex by cortical electrical stimulation study. Ann. Neurol. 54, 615-624.

Kahane, P., Minotti, L., Hoffmann, D., Lachaux, J. P., Ryvlin, P., Rosenow, F., and Lüders, H. O. (2004). "Invasive EEG in the definition of the seizure onset zone: depth electrodes," in Handbook of Clinical Neurophysiology, Vol. 3 eds F. Rosenow and H. Luders (Amsterdam: Elsevier BV), 109-133.

Kahane, P., Tassi, L., Francione, S., Hoffmann, D., Lo, R. G., and Munari, C. (1993). Electroclinical manifestations elicited by intracerebral electric stimulation "shocks" in temporal lobe epilepsy. Neurophysiol. Clin. 23, 305-326.

Kalitzin, S., Parra, J., Velis, D. N., and Lopes da Silva, F. H. (2002). Enhancement of phase clustering in the EEG/MEG gamma frequency band anticipates transitions to paroxysmal epileptiform activity in epileptic patients with known visual sensitivity. IEEE Trans. Biomed. Eng. 49, 1279-1286.

Kalitzin, S., Velis, D., Suffczynski, P., Parra, J., and da Silva, F. L. (2005). Electrical brain-stimulation paradigm for estimating the seizure onset site and the time to ictal transition in temporal lobe epilepsy. Clin. Neurophysiol. 116, 718-728.

Kalitzin, S. N., Velis, D. N., and da Silva, F.H. (2010). Stimulation-based anticipation and control of state transitions in the epileptic brain. Epilepsy Behav. $17,310-323$.

Kiss, Z. H., Anderson, T., Hansen, T., Kirstein, D., Suchowersky, O., and $\mathrm{Hu}$, B. (2003). Neural substrates of microstimulation-evoked tingling: a chronaxie study in human 
somatosensory thalamus. Eur. J. Neurosci. 18, 728-732.

Klingler, J., and Gloor, P. (1960). The connections of the amygdala and of the anterior temporal cortex in the human brain. J. Comp. Neurol. 115, 333-369.

Koch, U. R., Musshoff, U., Pannek, H. W., Ebner, A., Wolf, P., Speckmann, E. J., and Kohling, R. (2005). Intrinsic excitability, synaptic potentials, and short-term plasticity in human epileptic neocortex. J. Neurosci. Res. 80, 715-726.

Lachaux, J. P., Rudrauf, D., and Kahane, P. (2003). Intracranial EEG and human brain mapping. J. Physiol. Paris 97, 613-628.

Lacruz, M. E., Garcia Seoane, J. J., Valentin, A., Selway, R., and Alarcon, G. (2007). Frontal and temporal functional connections of the living human brain. Eur. J. Neurosci. 26, 1357-1370.

Landré, E., Turak, B., Toussaint, D., and Trottier, S. (2004). Intérêt des stimulations électriques intracérébrales en stéréo-électroencéphalographie dans les épilepsies partielles. Epilepsies 16, 213-225

Lee, H. W., Hong, S. B., Seo, D. W., Tae, W. S., and Hong, S. C. (2000). Mapping of functional organization in human visual cortex: electrical cortical stimulation. Neurology 54, 849-854.

Lesser, R. P., Arroyo, S., Crone, N., and Gordon, B. (1998). Motor and sensory mapping of the frontal and occipital lobes. Epilepsia 39(Suppl. 4), S69-S80.

Lesser, R. P., Lee, H. W., Webber, W. R., Prince, B., Crone, N.E., and Miglioretti, D. L. (2008). Short-term variations in response distribution to cortical stimulation. Brain 131, 1528-1539.

Lian, J., Bikson, M., Shuai, J., and Durand, D. M. (2001). Propagation of nonsynaptic epileptiform activity across a lesion in rat hippocampal slices. $J$. Physiol. 537, 191-199.

Lieb, J. P., Dasheiff, R. M., and Engel, J. Jr. (1991). Role of the frontal lobes in the propagation of mesial temporal lobe seizures. Epilepsia 32, 822-837.

Lim, S. H., Dinner, D. S., Pillay, P. K., Luders, H., Morris, H. H., Klem, G., Wyllie, E., and Awad, I. A. (1994). Functional anatomy of the human supplementary sensorimotor area: results of extraoperative electrical stimulation. Electroencephalogr. Clin. Neurophysiol. 91, 179-193.

Lobel, E., Kahane, P., Leonards, U., Grosbras, M., Lehericy, S., Le Bihan, D., and Berthoz,A. (2001). Localization of human frontal eye fields: anatomical and functional findings of functional magnetic resonance imaging and intracerebral electrical stimulation. $J$. Neurosurg. 95, 804-815.
Luders, H., Lesser, R. P., Hahn, J., Dinner, D. S., Morris, H., Resor, S., and Harrison, M. (1986). Basal temporal language area demonstrated by electrical stimulation. Neurology 36, 505-510.

Luders, H.O., Dinner, D. S., Morris, H.H., Wyllie, E., and Comair, Y. G. (1995). Cortical electrical stimulation in humans. The negative motor areas. Adv. Neurol. 67, 115-129.

Mandonnet, E., Winkler, P.A., and Duffau, H. (2010). Direct electrical stimulation as an input gate into brain functional networks: principles, advantages and limitations. Acta Neurochir. (Wien) 152, 185-193.

Mani, J., Diehl, B., Piao, Z., Schuele, S. S., Lapresto, E., Liu, P., Nair, D. R., Dinner, D. S., and Luders, H. O. (2008). Evidence for a basal temporal visual language center: cortical stimulation producing pure alexia. Neurology 71, 1621-1627.

Matsumoto, R., Nair, D. R., LaPresto, E., Bingaman, W., Shibasaki, H., and Luders, H. O. (2007). Functional connectivity in human cortical motor system: a cortico-cortical evoked potential study. Brain 130, 181-197.

Matsumoto, R., Nair, D. R., LaPresto, E., Najm, I., Bingaman, W., Shibasaki, H., and Luders, H. O. (2004). Functional connectivity in the human language system: a cortico-cortical evoked potential study. Brain 127, 2316-2330.

McIntyre, C. C., Savasta, M., Kerkerian-Le, G.L., and Vitek,J.L.(2004).Uncovering the mechanism(s) of action of deep brain stimulation: activation, inhibition, or both. Clin. Neurophysiol. 115, 1239-1248.

Merrill, D. R., Bikson, M., and Jefferys, J. G. (2005). Electrical stimulation of excitable tissue: design of efficacious and safe protocols. J. Neurosci. Methods 141, 171-198.

Mesulam, M. M. (1979). Tracing neural connections of human brain with selective silver impregnation. Observations on geniculocalcarine, spinothalamic, and entorhinal pathways. Arch. Neurol. 36, 814-818.

Miesenbock, G. (2009). The optogenetic catechism. Science 326, 395-399.

Morimoto, K., Fahnestock, M., and Racine, R. J. (2004). Kindling and status epilepticus models of epilepsy: rewiring the brain. Prog. Neurobiol. 73, 1-60.

Mulak, A., Kahane, P., Hoffmann, D., Minotti, L., and Bonaz, B. (2008). Brain mapping of digestive sensations elicited by cortical electrical stimulations. Neurogastroenterol. Motil. 20, 588-596.
Munari, C., Kahane, P., Tassi, L., Francione S., Hoffmann, D., Lo, R. G., and Benabid, A. L. (1993). Intracerebral low frequency electrical stimulation: a new tool for the definition of the "epileptogenic area"? Acta Neurochir. Suppl. (Wien) 58, 181-185.

Nathan, S. S., Sinha, S. R., Gordon, B. Lesser, R. P., and Thakor, N. V. (1993). Determination of current density distributions generated by electrical stimulation of the human cerebral cortex. Electroencephalogr. Clin. Neurophysiol. 86, 183-192.

Nowak, L. G., and Bullier, J. (1998a). Axons, but not cell bodies, are activated by electrical stimulation in cortical gray matter. I. Evidence from chronaxie measurements. Exp. Brain Res. 118, 477-488.

Nowak, L. G., and Bullier, J. (1998b). Axons, but not cell bodies, are activated by electrical stimulation in cortical gray matter. II. Evidence from selective inactivation of cell bodies and axon initial segments. Exp. Brain Res. 118, 489-500.

Ojemann, G.A. (2003). The neurobiology of language and verbal memory: observations from awake neurosurgery. Int J. Psychophysiol. 48, 141-146.

Parra, J., Kalitzin, S. N., Iriarte, J., Blanes, W., Velis, D. N., and Lopes da Silva, F. H. (2003). Gamma-band phase clustering and photosensitivity: is there an underlying mechanism common to photosensitive epilepsy and visua perception? Brain 126, 1164-1172.

Paus, T., Jech, R., Thompson, C. J., Comeau, R., Peters, T., and Evans, A. C. (1997). Transcranial magnetic stimulation during positron emission tomography: a new method for studying connectivity of the human cerebral cortex. J. Neurosci. 17, 3178-3184.

Penfield, W., and Boldrey, E. (1937) Somatic motor and sensory representation in the cerebral cortex of man as studied by electrical stimulation. Brain 60, 389-443

Penfield, W. G., and Jasper, H. H. (1954) Epilepsy and the Functional Anatomy of the Human Brain. Boston, MA: Little Brown \& Co.

Penny, W. D., Stephan, K. E., Mechelli, A., and Friston, K. J. (2004). Comparing dynamic causal models. Neuroimage 22, 1157-1172.

Piallat, B., Chabardes, S., Devergnas, A., Torres, N., Allain, M., Barrat, E., and Benabid, A. L. (2009). Monophasic but not biphasic pulses induce brain tissue damage during monopolar highfrequency deep brain stimulation. Neurosurgery 64, 156-162; discussion 162-153.

Puce, A., Allison, T., and McCarthy, G. (1999). Electrophysiological studies of human face perception. III: effects of top-down processing on facespecific potentials. Cereb. Cortex 9 445-458.

Quian Quiroga, R., Kraskov, A., Kreuz, T., and Grassberger, P. (2002). Performance of different synchronization measures in real data: a case study on electroencephalographic signals. Phys. Rev. E 65, 041903.

Rattay, F. (1999). The basic mechanism for the electrical stimulation of the nervous system. Neuroscience 89 , 335-346.

Reichova, I., and Sherman, S. M. (2004) Somatosensory corticothalamic projections: distinguishing drivers from modulators. J. Neurophysiol. 92, 2185-2197.

Roebroeck, A., Formisano, E., and Goebel, R. (2009). The identification of interacting networks in the brain using fMRI: model selection, causality and deconvolution. Neuroimage doi: 10.1016/j. neuroimage.2009.1009.1036.

Rosenberg, D. S., Mauguiere, F., Catenoix, H., Faillenot, I., and Magnin, M. (2009). Reciprocal thalamocortical connectivity of the medial pulvinar: a depth stimulation and evoked potential study in human brain. Cereb. Cortex 19, 1462-1473

Rosenberg, D. S., Mauguiere, F., Demarquay, G., Ryvlin, P., Isnard, J., Fischer, C., Guenot, M., and Magnin, M. (2006). Involvement of medial pulvinar thalamic nucleus in human temporal lobe seizures. Epilepsia 47, 98-107.

Saillet, S., Langlois, M., Feddersen, B., Minotti, L., Vercueil, L., Chabardes, S., David, O., Depaulis, A., Deransart, C., and Kahane, P. (2009). Manipulating the epileptic brain using stimulation: a review of experimental and clinical studies. Epileptic Disord. 11, 100-112.

Schiller, Y., and Bankirer, Y. (2007). Cellular mechanisms underlying antiepileptic effects of low- and high-frequency electrical stimulation in acute epilepsy in neocortical brain slices in vitro. J. Neurophysiol. 97, 1887-1902.

Schulz, R., Luders, H. O., Tuxhorn, I., Ebner, A., Holthausen, H., Hoppe, M., Noachtar, S., Pannek, H., May, T. and Wolf, P. (1997). Localization of epileptic auras induced on stimulation by subdural electrodes. Epilepsia 38, 1321-1329.

Selimbeyoglu, A., and Parvizi, J. (2010). Electrical stimulation of the human brain: perceptual and behavioral phenomena reported in the old and new literature. Front. Hum. Neurosci. 4:46. doi: 10.3389/fnhum.2010.00046

Sharp, D. J., Scott, S. K., and Wise, R. J. (2004). Retrieving meaning after 
temporal lobe infarction: the role of the basal language area. Ann. Neurol. 56, 836-846.

Sinai, A., Crone, N. E., Wied, H. M., Franaszczuk, P. J., Miglioretti, D., and Boatman-Reich, D. (2009). Intracranial mapping of auditory perception: event-related responses and electrocortical stimulation. Clin. Neurophysiol. 120, 140-149.

Spencer, S. S., Sperling, M. R., Shewmon, D. A., and Kahane, P. (2006). "Intracranial electrodes," in Epilepsy: A Comprehensive Textbook, eds J. Engel Jr. and T. A. Pedley (Philadelphia: Lippincott Williams \& Wilkins), 1791-1815.

Sporns, O., Tononi, G., and Kotter, R. (2005). The human connectome: a structural description of the human brain. PLoS Comput. Biol. 1:e42. doi: 10.1371/journal.pcbi.0010042.

Steriade, M., Jones, E. G., and McCormick, D. A. (1997). Thalamus Vol. I: Organisation and Function. Amsterdam: Elsevier.

Suffczynski, P., Kalitzin, S., da Silva, F. L., Parra, J., Velis, D., and Wendling, F. (2008). Active paradigms of seizure anticipation: computer model evidence for necessity of stimulation. Phys. Rev. E. Stat. Nonlin. Soft Matter Phys. 78, 051917.

Sumner, P., Nachev, P., Morris, P., Peters, A. M., Jackson, S. R., Kennard, C., and Husain, M. (2007). Human medial frontal cortex mediates unconscious inhibition of voluntary action. Neuron 54, 697-711.

Szelenyi, A., Kothbauer, K., de Camargo, A. B., Langer, D., Flamm, E. S., and Deletis, V. (2005). Motor evoked potential monitoring during cerebral aneurysm surgery: technical aspects and comparison of transcranial and direct cortical stimulation. Neurosurgery 57, 331-338; discussion 331-338.
Thiebaut de Schotten, M., Urbanski, M., Duffau, H., Volle, E., Levy, R., Dubois, B., and Bartolomeo, P. (2005). Direct evidence for a parietal-frontal pathway subserving spatial awareness in humans. Science 309, 2226-2228.

Tolias, A. S., Sultan, F., Augath, M., Oeltermann, A., Tehovnik, E. J., Schiller, P. H., and Logothetis, N. K. (2005). Mapping cortical activity elicited with electrical microstimulation using FMRI in the macaque. Neuron 48, 901-911.

Trebuchon-Da Fonseca, A., Guedj, E., Alario, F. X., Laguitton, V., Mundler, O., Chauvel, P., and Liegeois-Chauvel, C. (2009). Brain regions underlying word finding difficulties in temporal lobe epilepsy. Brain 132, 2772-2784.

Tsuda, I. (2001). Toward an interpretation of dynamic neural activity in terms of chaotic dynamical systems. Behav. Brain Sci. 24, 793-810.

Valentin, A., Alarcon, G., Garcia-Seoane, J. J., Lacruz, M. E., Nayak, S. D., Honavar, M., Selway, R. P., Binnie, C. D., and Polkey, C. E. (2005a). Single-pulse electrical stimulation identifies epileptogenic frontal cortex in the human brain. Neurology 65, 426-435.

Valentin, A., Alarcon, G., Honavar, M., Garcia Seoane, J. J., Selway, R. P., Polkey, C. E., and Binnie, C. D. (2005b). Single pulse electrical stimulation for identification of structural abnormalities and prediction of seizure outcome after epilepsy surgery: a prospective study. Lancet Neurol. 4, 718-726.

Valentin, A., Anderson, M., Alarcon, G., Seoane, J. J., Selway, R., Binnie, C. D., and Polkey, C. E. (2002). Responses to single pulse electrical stimulation identify epileptogenesis in the human brain in vivo. Brain 125, 1709-1718.

Varela, F., Lachaux, J. P., Rodriguez, E., and Martinerie, J. (2001). The brainweb: phase synchronization and large-scale integration. Nat. Rev. Neurosci. 2, 229-239.

Varela, F. G., Maturana, H. R., and Uribe, R. (1974). Autopoiesis: the organization of living systems, its characterization and a model. Curr. Mod. Biol. 5, 187-196.

Vignal, J. P., Maillard, L., McGonigal, A., and Chauvel, P. (2007). The dreamy state: hallucinations of autobiographic memory evoked by temporal lobe stimulations and seizures. Brain 130, 88-99.

Wendling, F., Ansari-Asl, K., Bartolomei, F., and Senhadji, L. (2009). From EEG signals to brain connectivity: a modelbased evaluation of interdependence measures. J. Neurosci. Methods 183, 9-18.

Wieser, H. G., Bancaud, J., Talairach, J., Bonis, A., and Szikla, G. (1979). Comparative value of spontaneous and chemically and electrically induced seizures in establishing the lateralization of temporal lobe seizures. Epilepsia 20, 47-59.

Wilson, C. L., Isokawa, M., Babb, T. L., and Crandall, P.H. (1990). Functional connections in the human temporal lobe. I. Analysis of limbic system pathways using neuronal responses evoked by electrical stimulation. Exp. Brain Res. 82, 279-292.

Wilson, C. L., Isokawa, M., Babb, T. L., Crandall, P. H., Levesque, M. F., and Engel, J. Jr. (1991). Functional connections in the human temporal lobe. II. Evidence for a loss of functional linkage between contralateral limbic structures. Exp. Brain Res. 85, 174-187.

Wilson, C. L., Khan, S. U., Engel, J. Jr., Isokawa, M., Babb, T. L., and Behnke, E. J. (1998). Paired pulse suppression and facilitation in human epileptogenic hippocampal formation. Epilepsy Res. 31, 211-230.

Zangaladze, A., Sharan, A., Evans, J., Wyeth, D. H., Wyeth, E. G., Tracy,
J. I., Chervoneva, I., and Sperling, M. R. (2008). The effectiveness of low-frequency stimulation for mapping cortical function. Epilepsia 49, 481-487.

Zonenshayn, M., Mogilner, A. Y., and Rezai, A. R. (2000). Neurostimulation and functional brain imaging. Neurol. Res. 22, 318-325.

Zucker, R. S., and Regehr, W. G. (2002). Short-term synaptic plasticity. Annu. Rev. Physiol. 64, 355-405.

Zumsteg, D., Lozano, A. M., and Wennberg, R. A. (2006a). Depth electrode recorded cerebral responses with deep brain stimulation of the anterior thalamus for epilepsy. Clin. Neurophysiol. 117, 1602-1609.

Zumsteg, D., Lozano, A. M., Wieser, H. G., and Wennberg, R.A. (2006b). Cortical activation with deep brain stimulation of the anterior thalamus for epilepsy. Clin. Neurophysiol. 117, 192-207.

Conflict of Interest Statement: The authors declare that the research was conducted in the absence of any commercial or financial relationships that could be construed as a potential conflict of interest.

Received: 11 June 2010; accepted: 20 September 2010; published online: 20 October 2010.

Citation: David O, Bastin J, Chabardès S, Minotti L and Kahane P (2010) Studying network mechanisms using intracranial stimulation in epileptic patients. Front. Syst. Neurosci. 4:148. doi: 10.3389/ fnsys.2010.00148

Copyright (c) 2010 David, Bastin, Chabardès, Minottiand Kahane. This is an open-access article subject to an exclusive license agreement between the authors and the Frontiers Research Foundation, which permits unrestricted use, distribution, and reproduction in any medium, provided the original authors and source are credited. 